\title{
CORRIGENDUM
}

\section{Atomistic simulations of spinodal phase separation preceding polymer crystallization}

Richard H. Gee, Naida Lacevic and Laurence E. Fried

Nature Materials 5, 39-43 (2006); published online 25 December 2005; corrected after print 23 January 2009

In the above Letter, the parameters for the nonpolar polymer model used in the simulations were incorrectly specified as being identical to the polyethylene model of Paul et al. (ref. 21 of the Letter). However, the potential used in the Letter differed from that of Paul et al. in that the two-fold term in the C-C-C-C torsion was changed from $k_{\Phi}^{2}(1-\cos 2 \Phi)$, used in the polyethylene (PE) polymer model of Paul et al. to $k_{\Phi}^{2}(1+\cos 2 \Phi)$. 